\title{
Miomas Submucosos: Classificação Pré-Operatória para Avaliação da Viabilidade da Cirurgia Histeroscópica
}

\author{
Submucous Fibroids: Presurgical Classification to Evaluate the Viability \\ of Hysteroscopic Surgical Treatment
}

\begin{abstract}
Ricardo Bassil Lasmar ${ }^{1,2}$, Paulo Roberto Mussel Barrozo ${ }^{1}$, Rogério Dias ${ }^{1}$, Marco Aurélio Pinho de Oliveira ${ }^{2}$, Anaglória Pontes ${ }^{1}$, Daniel Spadoto Dias ${ }^{3}$
\end{abstract}

\section{RESUM0}

Objetivo: desenvolver uma nova classificação pré-operatória dos miomas submucosos para avaliação da viabilidade e do grau de dificuldade da miomectomia histeroscópica.

Métodos: conduzimos um estudo onde quarenta e quatro pacientes foram submetidas a ressecção histeroscópica de 51 miomas submucosos. Foram considerados a possibilidade da ressecção total do mioma, o tempo cirúrgico, o balanço hídrico e a incidência de complicações. Os miomas foram classificados pela classificação da sociedade Européia de Cirurgia Endoscópica (CSECE) e pela Classificação Proposta (CP) pelo nosso grupo, que, além do grau de penetração do mioma no miométrio, adiciona como parâmetros a extensão da base do mioma em relação à parede do útero, o tamanho do nódulo em centímetros e a topografia na cavidade uterina. Para análise estatística foram usados o teste de Fisher, o teste t de Student e a análise de variância. Foi considerado estatisticamente significativo quando o valor de p-valor foi menor que 0,05 no teste bicaudal.

Resultados: em 47 miomas a cirurgia histeroscópica foi considerada completa. Não houve diferença significativa entre os três niveis $(0,1$ e 2) da CSECE. Pela CP, a diferença quanto ao número de cirurgias completas foi significativa $(p=0,001)$ entre os dois níveis (grupos I e II). A diferença da duração da cirurgia quando se compara as duas classificações foi significativa. Em relação ao balanço hídrico, apenas a CP mostrou diferenças entre os níveis ( $p=0,02)$.

Conclusões: a CP inclui mais dados sinalizadores das dificuldades da miomectomia histeroscópica do que a CSECE, atualmente em uso. Deve ser enfatizado que o número de miomectomias histeroscópicas usado para essa análise foi modesto, sendo interessante a avaliação do desempenho dessa classificação em séries maiores de casos.

PALAVRAS-CHAVE: Mioma. Miomectomia. Histeroscopia.

\section{Introdução}

Leiomiomas uterinos são os tumores mais freqüentes do trato genital feminino, clinicamen-

\footnotetext{
${ }^{1}$ Setor de Endoscopia Ginecológica, Disciplina de Ginecologia da Faculdade de Medicina de Botucatu - UNESP. Curso de Pós-Graduação em Ginecologia e Obstetrícia ${ }^{2}$ Disciplina de Ginecologia da Faculdade de Medicina da Universidade do Estado do Rio de Janeiro - RJ

${ }^{3}$ Faculdade de Medicina de São José do Rio Preto - FAMERP - SP Correspondência:

Ricardo Bassil Lasmar

Rua Voluntários da Pátria 126, Sala 602 - Botafogo

22270-010 - Rio de Janeiro - RJ

e-mail: ricardo@lasmar.com.br
}

te aparentes em $25 \%$ das pacientes ${ }^{1}$. Os miomas são achados em até $77 \%$ das autópsias quando técnicas refinadas são usadas ${ }^{2}$. A incidência é mais alta em mulheres na terceira e quarta décadas e a raça negra é considerada um fator de risco ${ }^{3}$.

Os miomas são, habitualmente, diagnosticados em pacientes no menacme, sendo pouco comum na puberdade e em mulheres na pós-menopausa, faixa etária em que há tendência à diminuição do tamanho.

Os miomas submucosos são os que provocam sintomas como a menorragia e a infertilidade com maior freqüência ${ }^{4}$. Nesses casos, a miomectomia histeroscópica é alternativa terapêutica 
para as pacientes com sangramento uterino anormal e/ou infertilidade e que precisam preservar o seu útero com o menor dano miometrial possível $^{5,6}$

A técnica da miomectomia histeroscópica sofreu modificações significativas com o passar dos anos; iniciou-se, em 1978, com o uso do ressectoscópio urológico e da corrente monopolar, quando Neuwirth ${ }^{7}$ realizou o fatiamento do mioma ${ }^{8}$. Após quase uma década, Goldrath ${ }^{9}$ descreveu uma técnica utilizando apenas pinças de apreensão. Mais recentemente, Lasmar e Barrozo ${ }^{10}$, fizeram o uso da técnica de mobilização direta sem secção prévia da pseudocápsula do tumor.

Wamsteker et al. ${ }^{11}$ publicaram estudo com 51 miomectomias histeroscópicas, concluindo que miomas com mais de metade de porção intramural só deveriam ser submetidos a uma abordagem cirúrgica por histeroscopia em casos muito bem selecionados, a fim de minimizar a necessidade de nova cirurgia. Desse estudo compreende-se a importância de se estadiarem os miomas submucosos quanto ao tamanho e à extensão no miométrio antes da realização da cirurgia. O objetivo, portanto, é buscar bom resultado pós-operatório, evitando-se operar os miomas que, potencialmente, não permitiriam uma ressecção completa. Porém, até o presente momento, nenhuma tentativa foi feita para classificar o grau de dificuldade da miomectomia histeroscópica. Como a miomectomia histeroscópica é, por vezes, cirurgia de alta complexidade, deve ter sua indicação corretamente avaliada no pré-operatório, objetivando minimizar a morbidade que, eventualmente, possa estar associada ao procedimento. Devem-se ponderar também os limites da técnica cirúrgica e a necessidade ou não do uso dos análogos do GnRH.

Com a finalidade de estabelecer o prognóstico cirúrgico e permitir a comparação válida dos resultados da miomectomia histeroscópica é fundamental a existência de uma classificação mais abrangente, que possa agrupar, adequadamente, os procedimentos com o mesmo grau de dificuldade, visto que a Classificação da Sociedade Européia de Cirurgia Endoscópica (CSECE) usa, apenas, o grau de penetração do mioma submucoso no miométrio ${ }^{12}$.

O objetivo do presente estudo foi elaborar uma classificação para a miomectomia histeroscópica baseada no grau de dificuldade técnica para a realização do procedimento, recomendando a conduta mais indicada em cada situação.

\section{Pacientes e Métodos}

Foi realizado estudo retrospectivo que incluiu 44 pacientes submetidas a 51 miomectomias video-histeroscópicas, realizadas no período de julho de 2001 a novembro de 2003.

Todas as pacientes tinham avaliação ultrasonográfica transvaginal e/ou por ressonância magnética nos últimos 6 meses, e 2 eram portadoras de mioma submucoso e com indicação cirúrgica devido a sangramento uterino excessivo e/ou infertilidade.

Após anamnese e exame físico as pacientes foram submetidas à histeroscopia para confirmação diagnóstica, registrando-se o número de miomas, a penetração no miométrio, a extensão da base do nódulo em relação à parede acometida, a localização em relação à parede uterina e o terço da cavidade onde se encontrava o mioma. Apesar de o tamanho do mioma ter sido avaliado pela histeroscopia, contribuiu, de maneira significativa, no valor final a ultra-sonografia ou a ressonância magnética. Nos casos em que o examinador não achava possivel a miomectomia histeroscópica, mesmo em dois tempos, as pacientes foram orientadas a procurar outro tipo de tratamento e foram excluídas deste estudo.

A classificação proposta no presente estudo teve como base a análise subjetiva dos parâmetros que tornavam a cirurgia mais dificil. Foram considerados quatro critérios: penetração do nódulo no miométrio, extensão da base do nódulo em relação à parede do útero, tamanho do nódulo e topografia.

Para avaliação da penetração do nódulo no miométrio utilizamos a mesma sistemática da CSECE, na qual o mioma que se encontra totalmente protuso na cavidade uterina recebe o escore 0 ; o que tem sua maior porção na cavidade uterina e menor porção no miométrio recebe o escore 1 e aquele que tem a sua maior parte no miométrio recebe o escore 2 (Figura 1a).

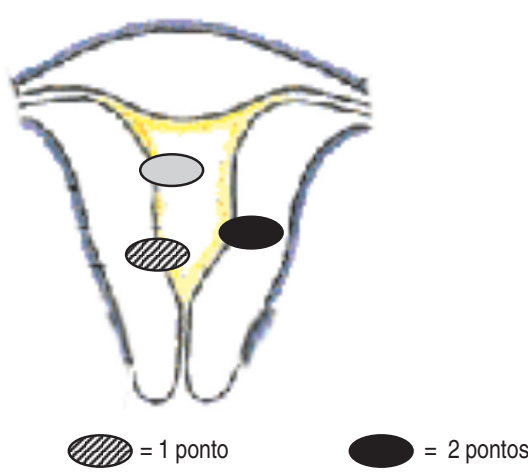

Figura 1a - Grau de penetração dos miomas 
A avaliação da extensão da base do nódulo em relação à parede do útero leva em consideração a parede em que está localizado o mioma, separando-a em ístmica, corporal e fúndica. Correlaciona-se a extensão da base do nódulo com a parede acometida e assim temos as seguintes possibilidades: quando o mioma acomete um terço ou menos da parede recebe o escore 0 ; quando a base do nódulo ocupa de um terço a dois terços da parede o escore é 1 e quando ele acomete mais de $2 / 3$ da parede tem o escore 2 (Figura $1 b$ ).

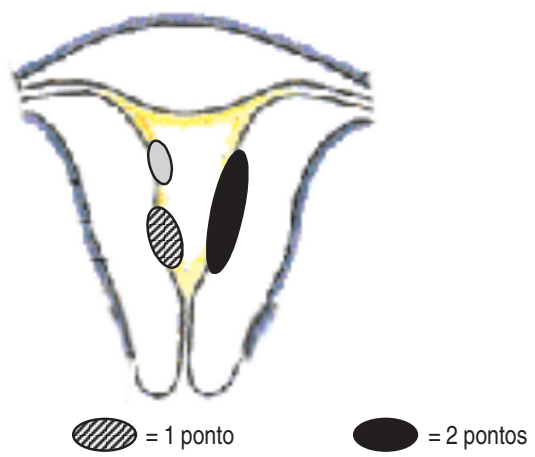

Figura 1b - Extensão da base do nódulo em relação a parede do útero

O tamanho considerado é o maior diâmetro encontrado em qualquer um dos exames de imagem: ultra-sonografia, histerossonografia ou ressonância magnética. Quando o nódulo mede $2 \mathrm{~cm}$ ou menos recebe o escore 0 ; se for maior que 2 $\mathrm{cm}$ e menor que $5 \mathrm{~cm}$ recebe o escore 1 , e medindo $5 \mathrm{~cm}$ ou mais, recebe o escore 2 .

A topografia é determinada pelo terço da cavidade uterina em que está situado o mioma, sendo escore 0 no terço inferior, escore 1 no terço médio e escore 2 no terço superior (Figura 1c). Quando o mioma está na parede lateral soma-se mais 1 ponto, independente do terço acometido (por ex., terço médio da parede lateral $=1+1$ ).

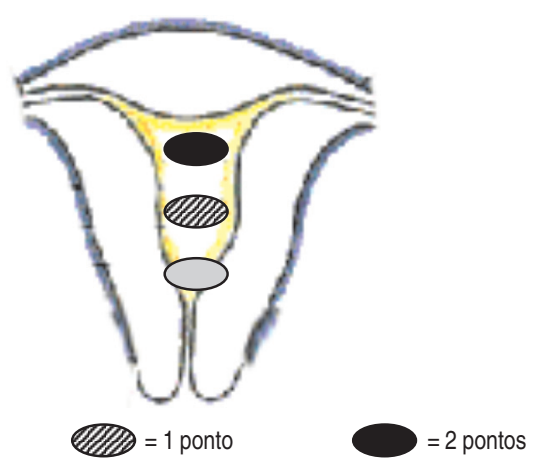

Figura 1c - Topografia (se o nódulo estiver na parede lateral, acrescenta-se 1 ponto)
A paciente pode apresentar mais de um mioma submucoso. Cada mioma submucoso tem sua pontuação individual (Tabela 1). Para enquadramento no grupo (I, II ou III) deve ser levado em consideração apenas o mioma que apresente o maior escore (Tabela 2). O escore total máximo é de nove pontos.

\begin{tabular}{lccccc}
\multicolumn{7}{c}{ Tabela 1 - Critérios empregados para a classificação de cada mioma. } \\
\hline \multicolumn{7}{c}{ Penetração } & Tamanho & Base & Terço & Parede lateral \\
\hline 0 & 0 & $=2 \mathrm{~cm}$ & $=1 / 3$ & inferior & +1 \\
1 & $=50 \%$ & $>2$ a $5 \mathrm{~cm}$ & $>1 / 3$ a $2 / 3$ & médio & +1 \\
2 & $>50 \%$ & $>5 \mathrm{~cm}$ & $>2 / 3$ & superior & +1 \\
Escore & + & + & + & + & $=$
\end{tabular}

Tabela 2 - Grupo no qual cada paciente se enquadra e conduta sugerida de acordo com o maior escore obtido.

\begin{tabular}{|c|c|c|}
\hline Escore & Grupo & Conduta sugerida \\
\hline 0 a 4 & I & Miomectomia histeroscópica com baixa complexidade \\
\hline 5 e 6 & $\|$ & $\begin{array}{l}\text { Miomectomia complexa, pensar em preparo com } \\
\text { análogo do GnRH e/ou cirurgia em } 2 \text { tempos }\end{array}$ \\
\hline 7 a 9 & III & Indicar outra técnica não histeroscópica \\
\hline
\end{tabular}

Na revisão do boletim operatório, foram anotadas as seguintes variáveis: ressecção completa ou incompleta (retirada de todo o mioma ou não), tempo operatório (em minutos), balanço hídrico (em mililitros) e as intercorrências.

As análises estatísticas das variáveis categóricas foram feitas com o uso das tabelas de contingência, optando-se pelo teste exato de Fisher. Para as variáveis numéricas foram usados o teste $t$ de Student para a comparação entre dois grupos e a análise de variância (ANOVA) para a comparação entre três grupos. Foi considerado estatisticamente significativo quando o valor de $\mathrm{p}$ foi menor que 0,05 no teste bicaudal.

Resultados

A indicação por infertilidade ou preocupação com gestação futura ocorreu em 15 casos e por sangramento uterino anormal em 29 casos. A idade variou de 28 a 78 anos, com média de 43,5 anos. O tempo operatório (não incluindo tempo de dilatação do colo e introdução do ressectoscópio) variou de 1,1 minuto a $38,4 \mathrm{mi}$ - 
nutos, com média de 11,2 minutos. O balanço hídrico (diferença entre o volume do meio líquido usado para a distensão do útero e o volume recuperado) variou de 0 a $1100 \mathrm{~mL}$, com uma média de 141,8 mililitros.

Segundo a CSECE, tivemos 8 miomas nível 0, 28 miomas nível 1 e 15 miomas nível 2 , ao passo que na Classificação Proposta (CP) 41 miomas foram classificados no grupo I e 10 miomas no grupo II.

Em quatro pacientes (8\%), a miomectomia foi considerada incompleta (CSECE = um nível $1 \mathrm{e}$ três níveis 2; $\mathrm{CP}=$ quatro do grupo II), sendo indicada uma segunda intervenção para a retirada do mioma.

$\mathrm{Na}$ análise das 47 miomectomias completas e classificadas pela CSECE observou-se sucesso de $100,0 \%(8 / 8)$ no nível 0; 96,4\% (27/28) para o nível 1 e $80,0 \%$ (12/15) para o nível 2, não sendo encontrada uma diferença significativa entre os grupos $(\mathrm{p}=0,18)$.

Fazendo-se a mesma análise mas considerando a CP observou-se sucesso de 100,0\% (41/ $41)$ no grupo I e 60,0\% (6/10) no grupo II. Essa diferença foi considerada significativa $(p=0,001)$.

Analisando-se o tempo operatório, na CSECE encontrou-se tempo médio de 5,4 minutos (variando de 1,3 a 13,8 min) no nível 0 , de 9,1minutos (1,1 a $33,2 \mathrm{~min})$ no nível 1 e de $18,1 \mathrm{~min}(1,3 \mathrm{a}$ $38,4)$ no nível 2. A diferença foi estatisticamente significativa $(\mathrm{p}=0,002)$. Na análise do tempo operatório na $\mathrm{CP}$ encontrou-se tempo médio de 8,2 minutos (1, 1 a $33 \mathrm{~min}$ ) no grupo I e de 23,2 minutos (11,7 a 38,4 min) no grupo II. A diferença foi estatisticamente significativa $(\mathrm{p}<0,0001)$.

Quanto ao balanço hídrico, na CSECE observou-se balanço hídrico médio de $56,2 \mathrm{~mL}$ no nível 0, de 123,2 mL no nível 1 e de $222 \mathrm{~mL}$ no nível 2 , não sendo encontrada diferença significativa entre os grupos $(p=0,17)$. Na CP observou-se balanço hídrico médio de $89,75 \mathrm{~mL}$ no grupo I e de $355 \mathrm{~mL}$ no grupo II, diferença que atingiu significância estatística $(\mathrm{p}=0,02)$.

Não foi possivel comparar as taxas de complicações entre as duas classificações, pois a incidência foi muito baixa (1 laceração de colo e 1 infecção urinária).

\section{Discussão}

A CSECE, usando apenas o nível de penetração do mioma no miométrio, não se mostrou muito eficaz para discriminar a complexidade da miomectomia histeroscópica. A divisão em três níveis
(0, 1 e 2) não auxiliou na predição do percentual de miomectomias completadas ou no grau de absorção de meio líquido (aferido pelo balanço hídrico). Apenas a diferença no tempo cirúrgico entre os três niveis foi significativa pela CSECE.

A CP no presente estudo teve melhor desempenho que a CSECE. As diferenças entre os três parâmetros analisados (número de miomectomias completas, tempo de cirurgia e balanço hídrico) se mostraram significativas quando a classificação inclui outros parâmetros além da penetração do mioma no miométrio. Observou-se que o tempo operatório médio é o dobro no nível 2 em relação ao nível 1 na CSECE, ao passo que quase que triplica do grupo I para o grupo II na CP. Além disso, o balanço hídrico médio é duplicado do nível 1 para o nível 2 da CSECE, ao passo que se eleva em praticamente 4 vezes do grupo I para o grupo II na CP.

Diante do exposto, acreditamos que a $\mathrm{CP}$ apresenta mais parâmetros sinalizadores das dificuldades da miomectomia histeroscópica do que a classificação da CSECE, atualmente em uso. Deve ser ressaltado que, embora o número de miomectomias histeroscópicas, do presente estudo, seja modesto e sabendo ser interessante e necessária avaliação do desempenho dessa classificação em séries maiores de casos, os autores acreditam que a $\mathrm{CP}$ vem trazer maiores subsídios em benefícios das indicações e realizações do procedimento cirúrgico, beneficiando, incontestavelmente, as pacientes.

\section{ABSTRACT}

Objective: to develop a new preoperative classification of submucous myomas to evaluate the viability and the degree of difficulty of hysteroscopic myomectomy.

Methods: forty-four patients were submitted to hysteroscopic resection of submucous myomas. The possibility of total resection of the myoma, the surgery duration, the fluid deficit, and the incidence of complications were evaluated. The myomas were classified by the Classification of the European Society of Endoscopic Surgery (CESES) and by the classification proposed $(C P)$ by our group, that besides the degree of penetration of the myoma in the myometrium, adds the parameters: extent of the base of the myoma as related to the uterine wall, the size of the myoma in centimeters and its topography at the uterine cavity. For statistical analysis the Fisher test, the Student $\mathrm{t}$ test and the analysis of variance were used. Statistic significance was considered when the pvalue was smaller than 0.05 in the bicaudal test.

Results: in 47 myomas the hysteroscopic surgery was considered complete. There was no significant difference among the three 
levels (0, 1 and 2) by CESES. By CP, the difference among the number of complete surgeries was significant $(p=0.001)$ between the two levels (groups I and II). The difference between the surgery duration was significant when the two classifications were compared. In relation to the fluid deficit, just $C P$ presented significant differences among the levels $(p=0,02)$.

Conclusions: the proposed classification includes more clues about the difficulties of the hysteroscopic myomectomy than the standard classification. It should be noted that the number of hysteroscopic myomectomies used for that analysis was modest, being interesting to evaluate the performance of the proposed classification in larger series of cases.

KEYWORDS: Myoma. Hysteroscopy. Myomectomy.

\section{Referências}

1. Buttram VC Jr, Reiter RC. Uterine leiomyomata: etiology, symptomatology and management. Fertil Steril 1981; 36:433-45

2. Cramer SF, Patel A. The frequency of uterine leiomyomas. Am J Clin Pathol 1990; 94:435-8.

3. Marshall LM, Spiegelman D, Barbieri RL, et al. Variation in the incidence of uterine leiomyoma among premenopausal women by age and race. Obstet Gynecol 1997; 90:967-73.
4. Pritts EA. Fibroids and infertility: a systematic review of the evidence. Obstet Gynecol Surv 2001; 56:483-91.

5. Narayan R, Goswamy K. Treatment of submucous fibroids, and outcome of assisted conception. J Am Assoc Gynecol Laparosc 1994; 1:307-11.

6. Hallez JP. Single-stage total hysteroscopic myomectomies: indications, techniques, and results. Fertil Steril 1995; 63:703-8.

7. Neuwirth RS. A new technique for and additional experience with hysteroscopic resection of submucous fibroids. Am J Obstet Gynecol 1978; $131: 91-4$

8. DeCherney A, Polan ML. Hysteroscopic management of intrauterine lesions and intractable uterine bleeding. Obstet Gynecol 1983; 61:392-7.

9. Goldrath MH. Vaginal removal of the pedunculated submucous myoma: the use of laminaria. Obstet Gynecol 1987; 70:670-2.

10.Lasmar R, Barrozo P. Histeroscopia: uma abordagem prática. $1^{\text {a }}$ ed. Rio de Janeiro: Medsi; 2002.

11.Wamsteker K, Emanuel MH, de Kruif JH. Transcervical hysteroscopic resection of submucous fibroids for abnormal uterine bleeding: results regarding the degree of intramural extension. Obstet Gynecol 1993; 82:736-40.

12.Lasmar RB, Brandão JON, Rica RPS, Bohm KR. Miomectomia. In: Lasmar RB, Brandão JON, Rica RPS, Bohm KR (Eds). Tratado de videoendoscopia ginecológica. São Paulo: Ateneu; 2003. p.1003-20.

Recebido em: 7/7/2003 Aceito com modificações em: 19/4/2004

\section{publicacoes@febrasgo.org.br \\ febrasgo@febrasgo.org.br \\ s.}

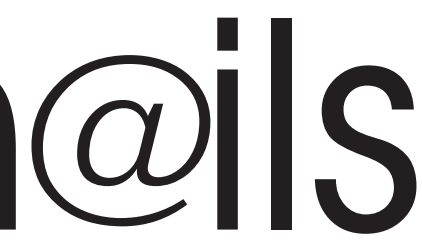

م0\%0 presidencia@febrasgo.org.br N0\% habilitacao@febrasgo.org.br $\mathrm{nO}_{\mathrm{N}}$ tego@febrasgo.org.br

\section{DÚVIDAS - SUGESTÕES - ESCLARECIMENTOS MANDE SEU E-MAIL}

